\title{
Requirements for Integrated Planning of Multi-Energy Systems
}

\author{
Nicolas Thie, Marco Franken, \\ Henrik Schwaeppe, Luis Böttcher, \\ Christoph Müller, Albert Moser \\ RWTH Aachen University \\ Aachen, Germany \\ n.thie@iaew.rwth-aachen.de
Matteo Pozzi, Angelo Gordini OPTIT s.r.l
Bologna, Italy
matteo.pozzi@optit.net

\author{
Klemens Schumann \\ Fraunhofer FIT \\ Munich, Germany \\ klemens.schumann@fit.fraunhofer.de
Kemal Burak Cakirer, Burcin Acan OEDAS
Eskişehir, Turkey
burak.cakirer@oedas.com.tr

\author{
Daniele Vigo, Michele Monaci, \\ Paolo Paronuzzi, Antonio Punzo \\ Alma Mater Studiorum - Università di \\ Bologna \\ Bologna, Italy \\ daniele.vigo@unibo.it
}

\author{
Umberto Desideri, Aldo Bischi \\ Università di Pisa \\ Pisa, Italy \\ umberto.desideri@unipi.it
}

\begin{abstract}
The successful realization of the climate goals agreed upon in the European Union's COP21 commitments makes a fundamental change of the European energy system necessary. In particular, for a reduction of greenhouse gas emissions over $80 \%$, the use of renewable energies must be increased not only in the electricity sector but also across all energy sectors, such as heat and mobility. Furthermore, a progressive integration of renewable energies increases the risk of congestions in the transmission grid and makes network expansion necessary. An efficient planning for future energy systems must comprise the coupling of energy sectors as well as interdependencies of generation and transmission grid infrastructure. However, in traditional energy system planning, these aspects are considered as decoupled. Therefore, the project PlaMES develops an approach for integrated planning of multi-energy systems on a European scale. This paper aims at analyzing the model requirements and describing the modeling approach.
\end{abstract}

Keywords-energy sector coupling, energy system planning, integrated generation and grid expansion, multi-energy systems

\section{INTRODUCTION}

Disruptive structural developments are necessary to deliver on the European Union's COP21 commitments, as confirmed by the "Clean Energy for All Europeans" package [1]. Specific targets and measures are identified for the energy efficiency, renewable energy sources share, governance and the electricity market design that envisages an increased crossborder cooperation and mobilization of public and private investment. Providing European energy system planners with the means to develop efficient strategies to reach these goals is however associated with significant challenges. First, the electricity sector only accounts for about $30 \%$ of greenhouse gas (GHG) emissions. Therefore, reaching the climate goals requires the use of renewable energies not only in the electricity sector but also in other energy sectors, in particular in heat/cooling and mobility sector [2]. Second, planning of an efficient energy system must comprise both the generation technology mix as well as the electricity grid infrastructure to determine an overall efficient investment decision [3].

Therefore, this paper proposes an approach for an integrated planning tool for multi-energy systems in a European scale including the following two main features:

- Energy sector coupling (electricity, heat, mobility)

- Integrated optimization of technology mix and electricity grid infrastructure

(C) 2020 IEEE. Personal use of this material is permitted. Permission from IEEE must be obtained for all other uses, in any current or future media, including reprinting/republishing this material for advertising or promotional purposes, creating new collective works, for resale or redistribution to servers or lists, or reuse of any copyrighted component of this work in other works.

\section{A. Energy sector coupling}

The ambitious climate goals require the coupled consideration of multiple energy sectors to facilitate the necessary GHG-reductions. In the last decade, the share of renewable energies sources (RES) in gross electricity consumption has increased significantly, representing in 2017 more than $18 \%$ of the European electricity generation (excluding large hydro power) in Germany and many other countries [4]. At the same time, the share of renewable energies in heat and mobility applications has risen only slightly. The final energy consumption of heat and mobility accounts for a large share of total primary energy mostly supplied by fossil fuels. For the successful continuation of the energy transition and a reduction of $\mathrm{CO}_{2}$ emissions, the carbon footprint reduction of further sectors besides electricity is necessary. Sector coupling, through a substitution of heat and mobility energy demands by either electricity coming from renewable energy sources or green fuels that could be produced also with renewable energy, will have significant impact on the assumed generation. Furthermore, sector coupling represents an extra flexibility option enabling the possibility of electric load shifting, e.g. thermostatically controllable loads such as compressor heat pumps and chillers as well as long term/seasonal storage of electric energy, e.g. power-to-gas. By providing this additional flexibility, sector-coupling can help handling increasing shares of RES and by that guarantee stability of the electric grid managing renewables 'volatility while fulfilling electric, thermal and mobility needs.

\section{B. Integrated optimization of technology mix and electricity grid infrastructure}

The optimization of the energy transition comprises adjustments to both the cross-sectoral technology mix and grid infrastructure at the transmission and distribution levels. As an example, the progressive integration of renewable energies leads to an increasing need for transmission grid expansions [5]. For example, in Germany, the high penetration of onshore and offshore wind power plants in the northern part of the country causes bottlenecks on transmission lines that supply demand centers in the western and southern parts. On the other hand, grid expansion is currently facing social-political acceptance problems. For this reason, while planning the optimal technology mix of the future, grid constraints and a limit for grid expansion have to be considered. consumption, e.g. about $75 \%$ in Germany, and is currently 
An integrated planning approach exhibits a high level of complexity for the mathematical formulation and modeling and requires an extensive data basis. Nonetheless, the integrated planning of the future technology mix and infrastructure has the evident advantage of combining the three aspects from the "EU Energy Trilemma", namely environmental impacts, energy affordability and energy security, in one solution method thus enabling the overall evaluation of the resulting target system (s. Fig. 1).

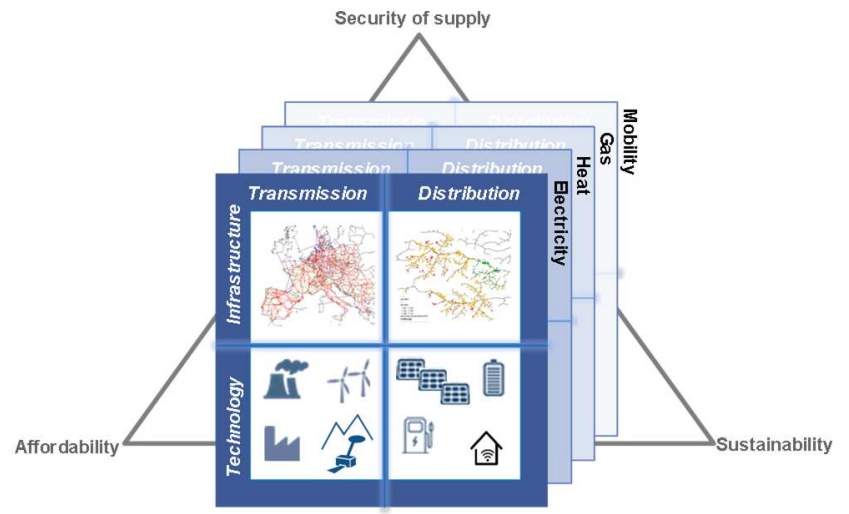

Fig. 1. Concept of the integrated PlaMES approach within the "EU Energy Trilemma".

\section{ANALYSIS OF THE STATE OF THE ART}

Traditionally, from both operational and planning perspectives energy sectors are considered to be decoupled from each other and models mainly focus on the electricity sector [6], [7], [8]. A multi-modal energy system approach allows a new perspective of energy system analysis by providing additional degrees of freedom to the system, which will lead to a technical, economic and ecological performance increase compared to traditionally decoupled energy systems, including:

- an increase in overall energy efficiency and therefore a reduction in primary energy consumption needed to fulfill electric, thermal and mobility needs;

- an optimal dispatch of central and distributed electric energy generation and storage units by optimal marketbased interactions;

- an increase in energy system flexibility, e.g. by the combination of heat pumps and power-to-heat storages or by a more flexible future power plant operation;

- a high $\mathrm{CO}_{2}$-reduction potential through the substitution of fossil energy carriers, directly by electricity from renewable energies or indirectly by synthetic gas, in the heat and mobility sectors.

- an increase in energy system flexibility increasing the overall electric system resilience towards extreme events, e.g. by the combination of power-to-heat systems such as heat pumps or electric heaters

A central question is the impact and the potential of energy sector coupling on the long-term achievement of climate goals [9], [10]. Approaches for the coupled consideration of energy sectors as well as the resulting effects on the operation and planning of energy systems are current issues in scientific work [11]. In addition, the integrated consideration of both centralized and distributed technologies and of different flexibility options is investigated [12].
Infrastructural planning challenges considering the optimization of generation technology mix and grid infrastructure are currently addressed in a decoupled fashion. Existing planning procedures and tools focus on the individual problems, e.g. the Ten Year Network Development Plan (TYNDP) provides a regularly updated European infrastructure development plan based on predefined scenarios [5]. The same happens at country level, as an example the german Grid Development Plan (GDP) addresses national electrical grid expansion measures also based on predefined scenarios (same for other national grid development plans). This is also true for the gas infrastructure for which a separate GDP is developed [13].

The combined consideration of a multi-energy technology mix optimization and grid expansion planning will lead to innovative solutions for the future energy system design how to tackle RES volatility and how to decarbonize the European energy system.

\section{METHODOLOGY}

The tool to be developed will model the investment decision, on the one hand, in energy supply structures in electricity, heat and mobility sectors and, on the other hand, in electricity grids on transmission and distribution level. The goal is to determine an efficient transition from the current system to an environmentally sustainable energy system (s. Fig. 2). The problem will be divided into two stages with increasing level of detail concerning the regional resolution.

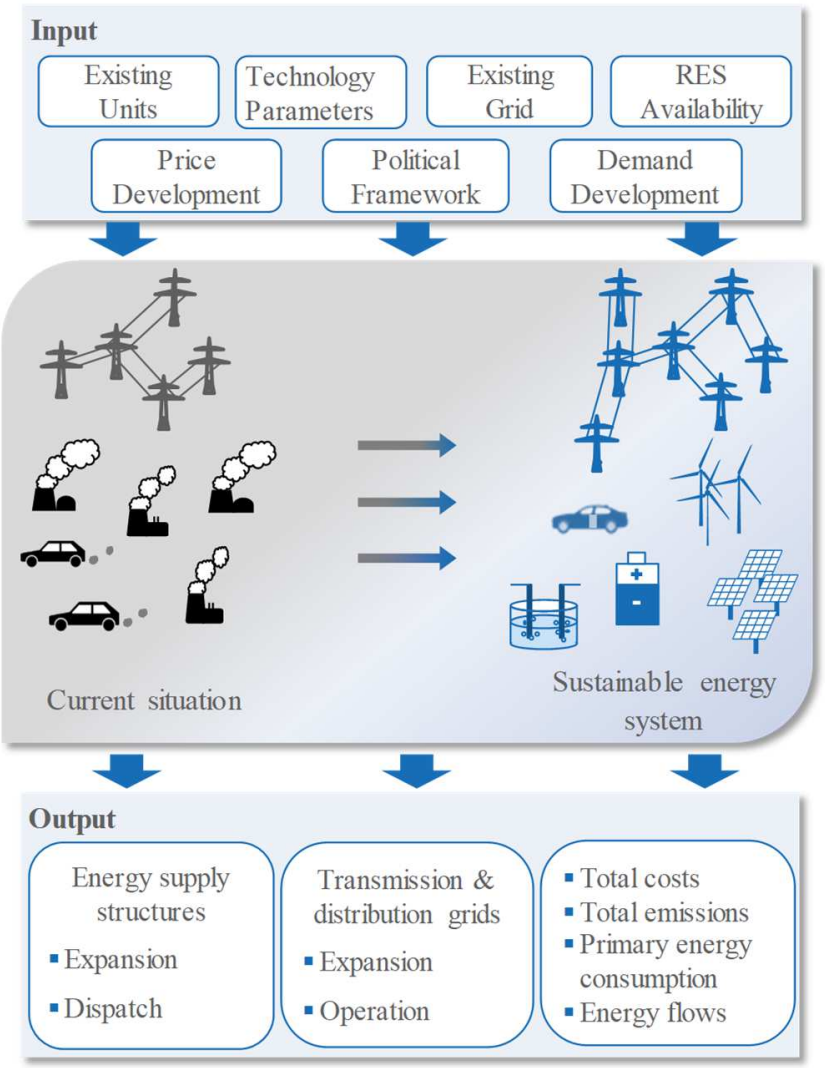

Fig. 2. Schematic of the planned methodology.

\section{A. First stage-European-wide energy system planning}

In a first stage, the European energy system is planned considering multiple energy sectors and transmission grid constraints. The planning is based on a scenario framework, which is an input for the model. The scenario framework 
originates from the status quo system, which includes existing grid infrastructure, generation units and demand. Furthermore, uncertain parameters like development of prices, political frameworks and consumption are included in the scenario and can be investigated via sensitivity analysis. As a first step of the system planning, the supply task for the future energy system is modeled based on the scenario framework.

\section{Modeling the supply task}

In countries where transmission grid data as well as demand data on electricity, heat and mobility and meteorological data is available in a regional resolution, the country is divided into smaller areas and planned in higher level of detail (focus region). In countries where regional resolved data is not available, technologies within the country are modelled in a more aggregated way (rest of Europe).

Within the focus region, the demand data on electricity, heat and mobility is generated by a bottom-up modeling approach [14]. Therefore, various data is necessary in a high granularity, e.g. postal code areas. To model household electricity and heat demand, socio-economic data is necessary. With information about the amount, age and size of buildings in each area [15], a representative database of all buildings for the country is created. Combined with information about the amount and average size of households per area [15], the size of each building in the database is defined. Load profiles are derived by combining the building database with standard heat and electrical demand patterns for various standard building types [16]. For modeling the electrical and heat demand of the sectors commercial trade service (CTS) and industry, information about the distribution of the companies and their size in each area is used [15]. By that, a database of the CTS and industry in each area of the focus region is generated. Considering standard load profiles and average demand per employee in each sector [17], the electrical and heat demand of those companies is defined.

The rest of Europe outside the focus region is modelled top-down. Available aggregated data concerning overall consumption for electricity and, if available, other sectors is used to determine the Europe-wide supply task.

\section{System Planning Model}

The system planning model is formulated as an optimization problem, which can be divided into objective function and optimization constraints.

The problem follows the objective function to minimize the overall system costs. This includes capital expenditures (CAPEX) for investment decisions as well as operational expenditures (OPEX) for system operation (dispatch).

To guarantee that the results of the planning tool are compliant with national and European climate goals, an explicit upper limit for $\mathrm{CO}_{2}$-emissions within the focus region and for whole Europe will be added to the mathematical model as a constraint. In addition, also a minimum share of renewable energies in gross electricity consumption and final energy consumption will be considered as a constraint. To ensure that all types of energy demands are met at all times and in all regions, hourly energy balancing constraints for electricity, heat/cold, gas and mobility are added to the model as well.

To consider restrictions in local electricity generation due to potential congestions in the transmission grid and to consider limitations in placement decisions of new generation units, transmission grid constraints will be modelled. To be compliant with current regulatory framework, N-1 security of transmission grid operation has to be ensured in the planning stage.

To identify the most efficient technologies for the longterm planning of the energy system, the assessment of those technologies as well as the quantification of their impact on the different energy sectors and on the overall system (costs) is necessary. As a result, the modelling of the dispatch decisions of existing and expanded technologies in form of a unit commitment is needed to determine the efficient investment decision (s. Fig. 3).

The dispatch decision of power plants and storages depends on dispatch decisions of previous time steps. These time-coupling constraints require the modelling of the dispatch decision in temporally coherent periods of observation (the consideration of single and non-coupled time steps is not sufficient). Especially the modelling of seasonal storages require the optimization of temporally coherent time periods.

Implementation of high level planning of energy supply structures

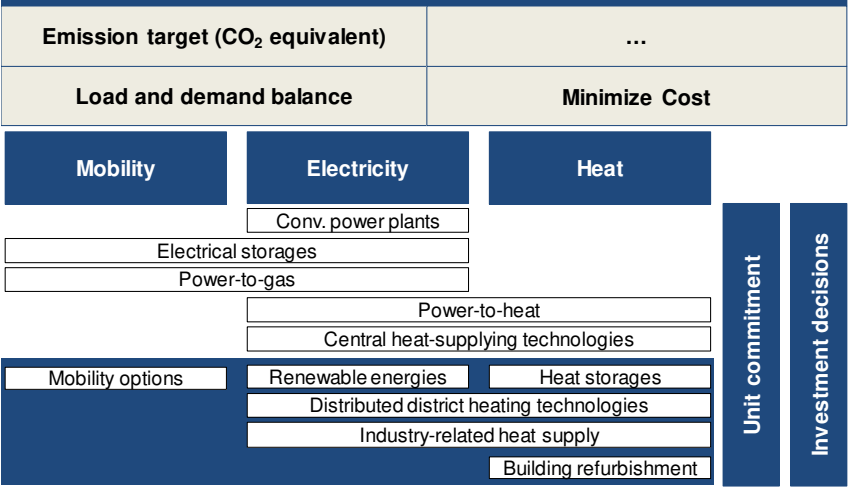

Fig. 3. Schenatic structure for planning the energy supply structures.

The modelling will include the following technologies:

- conventional (gas) power plants for electricity and heat supply

- electrical storages (e.g. pump hydro power plants, electrochemical battery storages)

- renewable energies for electricity generation (e.g. onshore wind turbines, offshore wind turbines, hydro power plants, biomass power plants, photovoltaics)

- distributed heat-supplying technologies (e.g. heat pumps, small scale combined heat and power (CHP), gas boilers, heating rods, biomass boilers, solar thermal etc.)

- heat supplying technologies in the industry (e.g. electric/gas boilers, high-temperature heat pumps, gas furnaces etc.)

- central heat-supplying technologies in district heating networks (e.g. large-scale heat pumps, electric boilers, gas boilers etc.)

- heat storages

- power-to-gas and power-to-fuel units (generation of hydrogen,synthetic methane and liquid fuels) 
- demand side management

- technologies in the mobility sector for passenger transport and freight transport (e.g. electric mobility, synthetic fuel cars etc.)

- technologies for transmission grid expansion: new High Voltage Alternating Current and Direct Current systems (HVAC systems and HVDC systems), voltage upgrades, High Temperature Low Sag (HTLS)upgrades, phase shifting transformers, etc.

Central generation and storage units will be modelled on single unit level, while distributed generation and storage units will be integrated in a simplified way via regional or nodal aggregation as regional sub problems. For this purpose, renewable energies will be modelled on e.g. postal code level considering local meteorological circumstances like wind velocities and solar radiation. Heat supply will be considered in an urban resolution since the range of heat transport by heating networks is limited to the order of magnitude of a few kilometers.

The consideration of power-to-heat technologies and CHP units on centralized and on distributed level enables the modelling of energy sector coupling between electricity and heat sectors. For CHP units, different fuel types could be used, like e.g. biogas, fossil gas or synthetic gas. Power-to-heat technologies involve e.g. heat pumps and heating rods in households as well as electric boilers, high-temperature heat pumps and electric furnaces in the industry. In addition, also central power-to-heat units like large-scale heat pumps in district heating networks will be considered. The conversion of electricity from renewable energies to heat by power-toheat technologies could contribute significantly in reducing use of fossil fuels in heat supply and could therefore help to reduce energy-related $\mathrm{CO}_{2}$-emissions.

The consideration of electric mobility in passenger and freight transport enables the modelling of energy sector coupling between electricity and mobility sectors. This includes private electric vehicles as well as electric busses. Depending on the driving behavior, resulting electricity demands are calculated, under consideration of the flexibility potential from battery storage systems. Again, the conversion of electricity from renewable energies to transport-energy by electric vehicles could substitute fossil fuels in the mobility sector and could therefore help to reduce energy-related $\mathrm{CO}_{2}$ emissions.

The consideration of power-to-gas (hydrogen production as well as methane production) and power-to-fuel technologies enables the modelling of energy sector coupling between electricity and gas/fuel sectors. Those technologies can be used to produce synthetic fuels out of renewable electricity. The produced synthetic fuels can be stored for long period, e.g. seasonal storage, and used later on in the heat and in the mobility sector (or even for backup electricity supply). Therefore, power-to-gas technology may provide on the one hand the possibility of seasonal storage helping to balance the electric grid and on the other hand contribute to the energy system decarbonization via "green fuel" production. To analyze the potential of power-to-gas technology, especially the temporal and spatial limitations of power generation from renewable energy sources as well as limitations in grid expansion due to social-political acceptance problems have to be considered.
In addition to transport capacities, also a simplified representation of costs for distribution grids shall be considered. A synthetic distribution grid model will provide cost functions relative to distribution grid expansion costs, which will be combined with the aggregated assets (distribution grid constraints cannot be considered in high detail in the first stage optimization, due to the aggregation of distributed technologies and the high level of complexity that would arise of an integrated consideration).

\section{B. Second stage - decentral energy system planning}

In a second stage, the regional results of the first stage planning tool are further broken down and planned in higher granularity on selected test cases. Such detailed granularity will be useful to find out shortcomings at distributed level which may influence the transmission level (e.g. solution from first stage is technically not feasible, or a feasible solution can just be achieved with very high costs). Feedback shall be given to the first stage planning tool and input parameters have to be iteratively adjusted or additional limitations have to be added (s. Fig. 4).

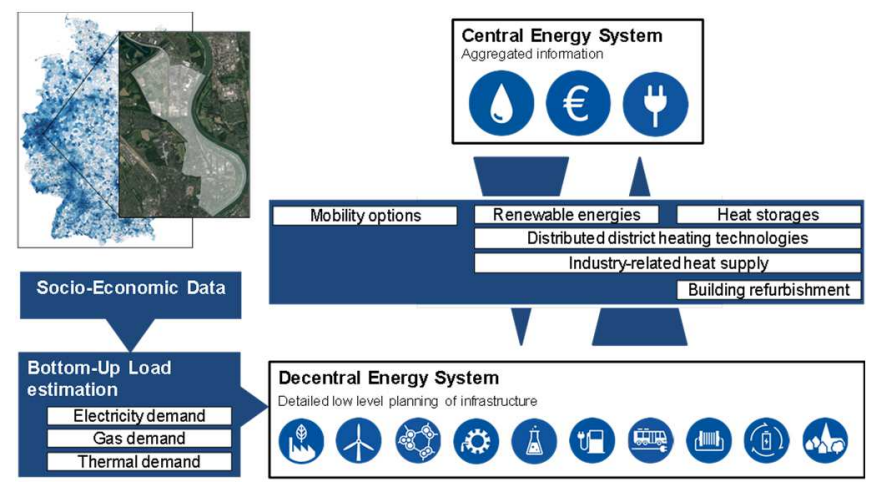

Fig. 4. Interdependency between central and decentral energy system planning.

Such regional optimal energy system design will allow to look with higher level of detail at the optimal integration of electric and gas network plus local district heating networks and mobility, both at local level, electric, and large distance transport, synthetically fueled. This will help local regional governments and multi-utilities to define the optimal roadmap for $\mathrm{CO}_{2}$-emissions reduction at national level and how the smart interaction of all regional results, with their exchanges of energy, will lead to a better global optimum at national and European level. Regional interactions as well as different energy sectors interactions (electricity, gas, heat and mobility) modeling and optimal design and management will enable to explore different objective functions and utilities marginal prices, developing new market configurations focusing on RES share increase, exploiting deep integration going from high voltage transmission to building level as well as testing different policies and exploring different stakeholders interests impact on the regional and up to the national level.

\section{Mathematical problem structure}

The modeling requirements lead to a complex non-linear and stochastic formulation, that will be most likely pragmatically reduced to more manageable sets of linear programming (LP) and and mixed integer linear programming (MILP) stochastic optimization problems. The structure of the problem is classified by the following aspects: 


\section{Decision variables}

Hourly operational decisions: Decision variables for dispatch decisions will be (mostly) continuous and plants characteristics curves assumed to be linear or piecewise linearized if further accuracy needed. It should be part of the discussion if it is possible to model the unit commitment of power plants using also binary variables for on/off states and therefore a mixed-integer linear approach for power plant operation.

Expansion decisions: For technologies that are modelled on single unit level (like e.g. power plants) as well as transmission lines the expansion decisions should be modelled as binary variables. For distributed technologies that are modelled in a more aggregated approach in regional resolution, the expansion decisions are continuous variables (like e.g. renewable generation units).

As some variables are continuous and some variables are binary variables, the overall problem will result in a mixedinteger optimization problem.

\section{System-coupling constraints}

Constraints to model climate goals: Constraints for an explicit upper limit for $\mathrm{CO}_{2}$-emissions and a minimum share of renewable energies in gross electricity consumption and final energy consumption will be integrated These linear constraints will couple all generation units and all time steps of the operational decision.

Energy balancing constraints: To ensure that all types of energy demands are met at all times, hourly energy balancing constraints for electricity, heat/cold, gas and mobility are added to the model. These linear constraints are formulated for each time step and will couple all generation and storage units and different energy sectors.

These constraints can be characterized as so-called complicating constraints. For large-scale problems, these constraints make it hard to solve the problem in closed manner. However, these constraints are usually good for decomposition, e.g. Lagrangian relaxation, Dantzig-Wolfe or Benders decomposition, due to the block-angular structure of the technology sub problems.

\section{Grid constraints}

Grid constraints (for electricity): Grid constraints for transmission and distribution grids shall be considered. In general, these constraints are non-linear. However, from an operational perspective, grid constraints at transmission level can be approximated by linearization approaches. To make these constraints linear e.g. linear sensitivities can be used, socalled Power Transfer Distribution Factors (PTDF) [18].

As part of the project, it will be discussed and analyzed if an integrated approach of grid constraints or an iterative approach between generation expansion planning and grid expansion planning is the more efficient solution method.

\section{Coupling of expansion decisions and operational decisions}

The consideration of constraints to couple expansion decisions and operational decisions of each unit is necessary. The operational constraints (without the expansion decision, see next bullet point "technical constraints of technologies") usually have a block-angular structure. Adding the expansion decisions, makes the problem more complicated [19].
The expansion variables can be characterized as so-called complicating variables. The resulting problem structure is usually good for decomposition, e.g. Benders decomposition (if the sub problem is a LP).

\section{Technical constraints of technologies (operational decisions)}

Technology specific constraints for the operational decisions will be considered. This includes e.g. ramping of generation units and storage continuity equations. These constraints result into a coupling of time steps within the operational problem.

In case of decomposing the expansion and the operational decisions (see "Coupling of expansion decisions and operational decisions"), the resulting operational sub problem can be solved in rolling time horizons to improve computational performance compared to a closed optimization over the whole time horizon [20]. To overcome these challenges, a combination of decomposition methods (e.g. Lagrangian Relaxation and Benders Decomposition) shall be analyzed to split the problem into smaller sub problems, which are solved iteratively. To overcome the problem of the non-linear structure of grid constraints, linear sensitivities for power flow calculation will be used.

\section{CASE STUdies}

Two case studies will be performed to show the adequacy and relevance of the developed modelling framework (s. Table I). In the second case study, a decentral energy system (e.g. one distribution grid area) will be planned based on the aggregated expansion targets defined in the central planning.

Case study 1: The first case study will comprise a Europewide system planning with the goal of reaching the overall climate goals up to 2050. Within the Europe-wide planning, a focus region will be defined which is investigated in more detail. This includes the question if the focus region can meet its own climate goals and how it can contribute in meeting the European climate goals. On central level, a special focus is laid on the impact of a limited amount of allowed transmission grid expansion on the optimal placement decisions of new generation and conversion units. In particular, the regional expansion of sector coupling units (i.e. power-to-gas and power-to-heat units) and the use of distributed flexibilities is analyzed.

Case study 2: On distributed level, the regional energy transition plan will be assessed based on the aggregated expansion targets determined by the central planning. Therefore, the main question of the decentral case study will be the integration of the new assets into the distribution grid area considering grid expansion measures as well as operational strategies. This includes generation capacities of renewable energy sources as well as technologies for flexible and local use of electricity from the heat and mobility sector (i.e. power-to-heat and electric-mobility).

The local case will be calculated within the boundaries provided by the central planning while, at the same time, it will provide boundary conditions to the central planning optimization problem. Results will highlight conflicting objectives between central and decentral level optimization such as conflicts between local and national actors regarding the energy use in different sectors and the emission reduction targets. 
TABLE I. Planned Use CASes

\begin{tabular}{|c|c|}
\hline Case Study 1 (central) & Case Study 2 (decentral) \\
\hline $\begin{array}{l}\text { Goal: } \\
\text { Meeting the European } 2050 \\
\text { climate goals by means of efficient } \\
\text { generation and grid expansion. }\end{array}$ & $\begin{array}{l}\text { Goal: } \\
\text { Detailed planning of a distribution } \\
\text { grid area with a high share of } \\
\text { renewable energies }\end{array}$ \\
\hline $\begin{array}{l}\text { Focus: } \\
\text { - Central energy system } \\
\text { planning on extra high voltage } \\
\text { level } \\
\text { - Renewable energy expansion } \\
\text { and locations } \\
\text { - future key technologies }\end{array}$ & $\begin{array}{l}\text { Focus: } \\
\text { - Distributed energy system } \\
\text { planning on mediul } \\
\text { voltage/low voltage level } \\
\text { - Flexibility options to support } \\
\text { grid operation } \\
\text { - Network expansion measures }\end{array}$ \\
\hline $\begin{array}{l}\text { Challenge: } \\
\text { - (Partial) phase out of coal and } \\
\text { nuclear power } \\
\text { - Congestions due to increasing } \\
\text { energy transport problem } \\
\text { - energy sector coupling }\end{array}$ & $\begin{array}{l}\text { Challenge: } \\
\text { - Integration of renewable } \\
\text { generation and flexibilities } \\
\text { within the distribution grid } \\
\text { - Distribution grid expansion to } \\
\text { guarantee security of supply }\end{array}$ \\
\hline $\begin{array}{l}\text { Dedicated user: } \\
\text { - Technology providers } \\
\text { (e.g portfolio planner) } \\
\text { - Policy makers }\end{array}$ & $\begin{array}{l}\text { Dedicated user: } \\
\text { - Distribution system operators } \\
\text { - Technology providers } \\
\text { (e.g. flexibilities) }\end{array}$ \\
\hline
\end{tabular}

On the one hand, the two case studies will allow for final validation of the tool functionalities at central and decentral planning level. On the other hand, the results will allow dedicated users to deduct first recommendations for facilitating an efficient development of the future energy system

\section{CONCLUSION}

This paper proposes a modeling approach for an integrated energy system planning aiming at reaching the European climate goals. In contrast to traditional planning approaches, multiple energy sectors are considered as coupled, which allows for substituting conventional, carbon-intensive energies not only in the electricity but also in other energy sectors. Furthermore, the model pursues an integrated optimization of generation and grid infrastructure expansion. This allows for determining an efficient trade-off between both and, hence, minimising the overall system costs.

\section{ACKNOWLEDGMENT}

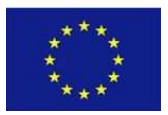
This research is part of the project PlaMES (Integrated Planning of Multi-Energy Systems). PlaMES has received funding from the European Union's Horizon 2020 research and innovation programme under grant agreement No 863922. The content of this deliverable reflects only the authors' view. The Innovation and Networks Executive Agency (INEA) is not responsible for any use that may be made of the information it contains.

\section{REFERENCES}

[1] European Commission, Clean Energy for all Europeans, Brussels, 2019.

[2] IRENA, Global Energy Transformation - A Roadmap to 2050, Abu Dhabi, 2018.

[3] R. Hemmati, R. Hooshmand, A. Khodabakhshian, Comprehensive review of generation and transmission expansion planning, IET Generation, Transmission \& Distribution, 2013.

[4] BP, Statistical Review of World Energy, $67^{\text {th }}$ Edition, London, 2018.

[5] ENTSOE, Ten Year Network Development Plan 2018, Brussels, 2019.

[6] G. Conzelmann, G. Boyd, V. Koritarov, T. Veselka, Multi-Agend Power Market Simulation using EMCAS, IEEE Power Engineering Society General Meeting, Volume 3, pp. 2829-2834, 2005.

[7] B. Özalay, Multikriterielles Verfahren zur Ausbauplanung und Regionalisierung zukünftiger Stromerzeugungsstrukturen, $\mathrm{PhD}$ thesis, Aachen, 2015.

[8] E. Guelpa, A. Bischi, V. Verda, M. Chertkov, H. Lund, Towards future infrastructures for sustainable multi-energy systems: A review, Energy, Vol. 184, pp: 2-21, 2019.

[9] Reflex - Analysis of the European Energy System, reserch project, URL: https://reflex-project.eu.

[10] SEEE. Sector coupling and European Integration in a Developing Energy System, research project, URL: https://www.diw.de/en/diw_01.c.523367.en/projects/sector_coupling and_european_integration_in_a_developing_energy_system.html.

[11] Plan4Res, research project, URL: https://www.plan4res.eu/.

[12] SmILES, Smart Integration of Energy Storages in Local Multi Energy Systems for maximising the Share of Renewables in Europe's Energy Mix, research project, URL: https://cordis.europa.eu/project/id/ 730936.

[13] Grid Development Plan Power 2030, Version 2019.

[14] C. Müller, A. Hoffrichter, L. Wyrwoll, C. Schmitt, M. Trageser, T. Kulms et al., Modeling framework for planning and operation of multimodal energy systems in the case of Germany, Applied Energy, Volume 250, pp. 1132-1146, 2019.

[15] Digital Data Services (DDS): Postal code 8 Germany XXL data packages (2014), Karlsruhe, 2014.

[16] Loga, T.; Stein, B.; Diefenbach, N.: TABULA building typologies in 20 European countries - Making energy-related features of residential building stocks comparable, Energy and Buildings, Vol. 132, pp. 4-12, 2016.

[17] B. Schlomann, H. Kleeberger, B. Geiger, A. Pich, E. Gruber, Energieverbrauch des Sektors Gewerbe, Handel, Dienstleistungen (GHD) in Deutschland für die Jahre 2011 bis 2013, Karlsruhe, Munich, Nurnberg, 2015.

[18] M. Liu, G. Gross, Effectiveness of the Distribution Factor Approximations Used in Congetion Modeling, $14^{\text {th }}$ Power Systems Computation Conference, Sevilla, 2002.

[19] C. Elsido, A. Bischi, P. Silva, E. Martelli, Two-stage MINLP algorithm for the optimal synthesis and design of networks of CHP units, Energy, Vol. 121, pp: 403-426, 2017

[20] A. Bischi, L. Taccari, E. Martelli, E. Amaldi, G. Manzolini, P. Silva, S. Campanari, E. Macchi, A rolling-horizon optimization algorithm for the long term operational scheduling of cogeneration systems, Energy, Vol 184, pp: 73-90, 2019 